\title{
An Anisotropic Cosmic-Ray Enhancement Event on 07-June-2015: A Possible Origin
}

\author{
Agnieszka Gil ${ }^{1}$ (D) Gennady A. Kovaltsov ${ }^{2,3}$ (D) \\ Vladimir V. Mikhailov ${ }^{4}$ - Alexander Mishev $^{2,5}$ (D) \\ Stepan Poluianov ${ }^{2,5}$ (ID) Ilya G. Usoskin ${ }^{2,5}$ (D)
}

Received: 15 August 2018 / Accepted: 30 October 2018 / Published online: 19 November 2018

(C) The Author(s) 2018

\begin{abstract}
A usual event, called anisotropic cosmic-ray enhancement (ACRE), was observed as a small increase $(\leq 5 \%)$ in the count rates of polar neutron monitors during $12-19$ UT on 07 June 2015 . The enhancement was highly anisotropic, as detected only by neutron monitors with asymptotic directions in the southwest quadrant in geocentric solar ecliptic (GSE) coordinates. The estimated rigidity of the corresponding particles is $\leq 1 \mathrm{GV}$. No associated detectable increase was found in the space-borne data from the Geostationary Operational Environmental Satellite (GOES), the Energetic and Relativistic Nuclei and Electron (ERNE) on board the Solar and Heliospheric Observatory (SOHO), or the Payload for Antimatter Matter Exploration and Light-nuclei Astrophysics (PAMELA) instruments, whose sensitivity was not sufficient to detect the event. No solar energetic particles were present during that time interval. The heliospheric conditions were slightly disturbed, so that the interplanetary magnetic field strength gradually increased during the event, followed by an increase of the solar wind speed after the event. It is proposed that the event was related to a crossing of the boundary layer between two regions with different heliospheric parameters, with a strong gradient of low-rigidity $(<1 \mathrm{GV})$ particles. It was apparently similar to another cosmic-ray enhancement (e.g., on 22 June 2015) that is thought to have been caused by the local anisotropy of Forbush decreases, with the difference that in our case, the interplanetary disturbance was not observed at Earth, but passed by southward for this event.
\end{abstract}

Keywords Solar cosmic rays

\section{I.G. Usoskin}

ilya.usoskin@oulu.fi

1 Institute of Mathematics and Physics, Siedlce University, 08110 Siedlce, Poland

2 Space Climate Research Unit, University of Oulu, 90014 Oulu, Finland

3 Ioffe Physical-Technical Institute, Politekhnicheskaya 26, 194021 St. Petersburg, Russia

4 MEPhI, Kashirskoe Shosse 31, 115409, Moscow, Russia

5 Sodankylä Geophysical Observatory, University of Oulu, 90014 Oulu, Finland 


\section{Introduction}

Variations in galactic cosmic-ray (GCR) flux near Earth are ultimately driven by solar variability on different timescales (Vainio et al., 2009). The temporal evolution of the cosmic-ray flux near Earth is traditionally and continuously monitored by the global network of neutron monitors (NMs), which is in continuous operation since the early 1950s (e.g. Simpson, 2000). The interannual variability of GCRs is driven by the 11-year solar cycle in the solar magnetic field that modulates them in the heliosphere (Potgieter, 2013). This is observed as a slow roughly 11-year variability of the NM count rate with a magnitude of about $25 \%$ at polar NMs (e.g. Usoskin, Bazilevskaya, and Kovaltsov, 2011), with alternating flat and sharp tops of the cycles representing the 22-year magnetic polarity cycle (Jokipii and Levy, 1977). On shorter timescales, the flux of GCRs is sporadically suppressed by interplanetary transient events (shocks, corotating regions, magnetic clouds, etc.), which cause reductions in GCR intensity, known as Forbush decreases (e.g. Lockwood, 1971; Cane, 2000; Ahluwalia and Fikani, 2007). In addition to GCR variability, sporadic enhancements of NM count rates are caused by solar energetic particle (SEP) events when a bulk of particles can be accelerated during solar eruptive events, flares, and/or coronal mass ejections (CMEs) to energies of hundreds of MeV up to several GeV (e.g. Desai and Giacalone, 2016; Klein and Dalla, 2017). Such events, when recorded by the ground-based NMs, are called ground-level enhancements (GLEs) and typically occur several times per solar cycle (see the International GLE database at http://gle.oulu.fi). Sometimes, the count rates of NMs can slightly increase as the result of a transient reduction of the geomagnetic shielding (Kudela, Bučík, and Bobík, 2008; Mohanty et al., 2016), but this is only related to detectors located at low or medium latitudes. Moreover, slight temporal enhancements in NM count rates can be observed during Forbush decreases (e.g. Cane, 2000). Cosmic-ray flux was enhanced on 22 June 2015, which was observed at high energies (several tens of $\mathrm{GeV}$ ) by the global muon detector network during the active phase of a strong Forbush decrease and was speculated to be caused by a local anisotropic effect near the heliospheric current sheet in highly disturbed conditions (Munakata et al., 2018). Another apparently similar cosmic-ray enhancement was observed on 18 January 2017, whose origin is not known yet (Mangeard et al., 2017).

Here we report an event of highly anisotropic cosmic-ray enhancement (ACRE) that was first registered by the Dome C standard and bare NMs, conventionally denoted as DOMC and DOMB, respectively, located at the Central Antarctic Plateau (Poluianov et al., 2015; Usoskin et al., 2015) during 7 June 2015, between roughly 12 and 19 UT. In contrast to the event on 22 June 2015 mentioned above, it was not accompanied by a Forbush decrease or other heliospheric disturbances recorded on Earth and only affected the lowest detectable energies below $1 \mathrm{GeV}$. This was a moderate increase in NM count rate that was not associated with a SEP flux, it was very anisotropic and lasted for several hours. The event was a subject of an informal discussion during the 34th International Cosmic Ray Conference in the Hague (2015), but has not been studied in detail since then. We present a detailed analysis of the event as recorded by the worldwide NM network and space-borne instruments, considering also the corresponding interplanetary conditions, and conclude that it was caused by focused scattering of GCRs on an interplanetary disturbance that passed Earth by.

\section{Datasets}

\subsection{Neutron Monitors}

We have analyzed data of all available NMs for the period around the event, viz. 6 through 8 June 2015. Data were obtained from the European Neutron Monitor Data Base (NMDB: 
Figure 1 Five-minute averaged count rates of the NMs (see Table 1) for the period of 6 through 8 June 2015. The time interval corresponding to the event is hatched.

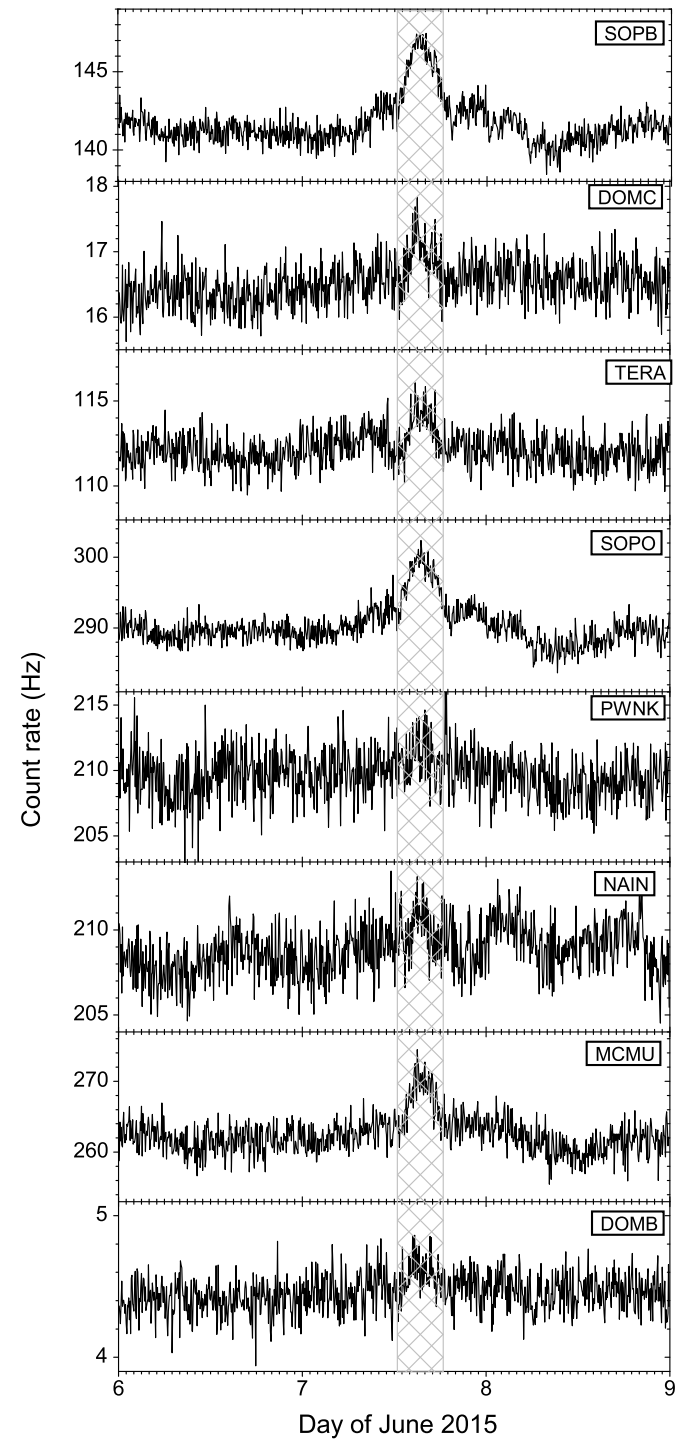

http://nmdb.eu, Mavromichalaki et al., 2011). Data from DOMC and DOMB are also available at http://cosmicrays.oulu.fi. These data are collected in the International GLE database (http://gle.oulu.fi). The time profiles of the count rates of the NMs, which depict an increase, are shown in Figure 1. A smooth increase in NM count rates between 12 and 19 UT on 7 June 2015 is visible, and the maximum is observed around 15 UT. The characteristics of the NMs and the recorded event magnitude are gathered in Table 1.

Figure 2 shows the same data as in Figure 1, but normalized to the maximum count rate during the peak of the event (15:00 UT on 07 June 2015). The strongest response of $5-8 \%$ was recorded by DOMB ("bare" or lead-free neutron monitor) and DOMC (standard NM), both located at the Condordia station on the Central Antarctic Plateau. Other Antarctic NMs, including South Pole, McMurdo, and Terre Adelie, recorded a statistically significant 
Table 1 Information on the detectors (NMs and the Payload for Antimatter Matter Exploration and LightNuclei Astrophysics (PAMELA) module): station name and acronym, effective vertical cutoff rigidity, $P_{\mathrm{c}}$ (in GV), height (in meters a.s.1.), geographical coordinates longitude and latitude, detector type, and the magnitude, $M$, of the event observed (in \%).

\begin{tabular}{llllllll}
\hline Name & Acronym & $P_{\mathrm{c}}$ & Height & Long. & Lat. & Type & $M$ \\
\hline Dome C & DOMC & $\approx 0$ & 3233 & $123.3 \mathrm{E}$ & $75.1 \mathrm{~S}$ & Mini-NM & $5-7 \%$ \\
Dome B & DOMB & $\approx 0$ & 3233 & $123.3 \mathrm{E}$ & $75.1 \mathrm{~S}$ & Bare & $6-8 \%$ \\
South Pole & SOPO & 0.1 & 2820 & & $90 \mathrm{~S}$ & $3 \mathrm{NM} 64^{*}$ & $3-4 \%$ \\
South Pole B & SOPB & 0.1 & 2820 & & $90 \mathrm{~S}$ & Bare & $3-4 \%$ \\
McMurdo & MCMD & $\approx 0$ & 48 & $166.6 \mathrm{E}$ & $77.9 \mathrm{~S}$ & $18 \mathrm{NM} 64$ & $3-4 \%$ \\
Terre Adelie & TERA & $\approx 0$ & 32 & $140 \mathrm{E}$ & $66.7 \mathrm{~S}$ & $9 \mathrm{NM} 64$ & $2-4 \%$ \\
Nain & NAIN & 0.3 & 46 & $61.7 \mathrm{~W}$ & $56.6 \mathrm{~N}$ & $18 \mathrm{NM} 64$ & $1-2 \%$ \\
Peawanuck & PWNK & 0.3 & 53 & $85.4 \mathrm{~W}$ & $55.0 \mathrm{~N}$ & $18 \mathrm{NM} 64$ & $\approx 1 \%$ \\
Barenzburg & BRBG & $\approx 0$ & 70 & $14.1 \mathrm{E}$ & $78.0 \mathrm{~N}$ & $18 \mathrm{NM} 64$ & $0^{\ddagger}$ \\
Thule & THUL & $\approx 0$ & 260 & $68.4 \mathrm{~W}$ & $76.6 \mathrm{~N}$ & $9 \mathrm{NM} 64$ & $0^{\ddagger}$ \\
Tixie Bay & TXBY & 0.3 & 0 & $128.9 \mathrm{E}$ & $71.6 \mathrm{~N}$ & $18 \mathrm{NM} 64$ & $0^{\ddagger}$ \\
Apatity & APTY & 0.5 & 177 & $33.3 \mathrm{E}$ & $67.6 \mathrm{~N}$ & $18 \mathrm{NM} 64$ & $0^{\ddagger}$ \\
Norilsk & NRLK & 0.6 & 0 & $88.05 \mathrm{E}$ & $69.3 \mathrm{~N}$ & $18 \mathrm{NM} 64$ & $0^{\ddagger}$ \\
Oulu & OULU & 0.7 & 15 & $25.5 \mathrm{E}$ & $65.1 \mathrm{~N}$ & $9 \mathrm{NM} 64$ & $0^{\ddagger}$ \\
Kerguelen & KERG & 1.1 & 0 & $70.3 \mathrm{E}$ & $49.4 \mathrm{~S}$ & $18 \mathrm{NM} 64$ & $0^{\ddagger}$ \\
\hline PAMELA & - & $\approx 1 \mathrm{GV}$ & $600 \mathrm{~km}$ & $24 \mathrm{~W}$ & $70 \mathrm{~S}$ & space & $\leq 1.5 \%$ \\
\hline
\end{tabular}

${ }^{\dagger}$ Parameters are given for the southernmost point of orbit at 14:34 UT.

* The SOPO NM consists of three 1NM64 units, which leads to a slightly decreased registration efficiency compared to the standard 3NM64 (Mangeard et al., 2016).

¥No distinguishable enhancement above the background.

Figure 2 Normalized smoothed count rates of the NMs ( $c f$. Figure 1). All curves were smoothed by the 45 -minute running average and normalized to unity on 15:00 UT 7 June 2015.

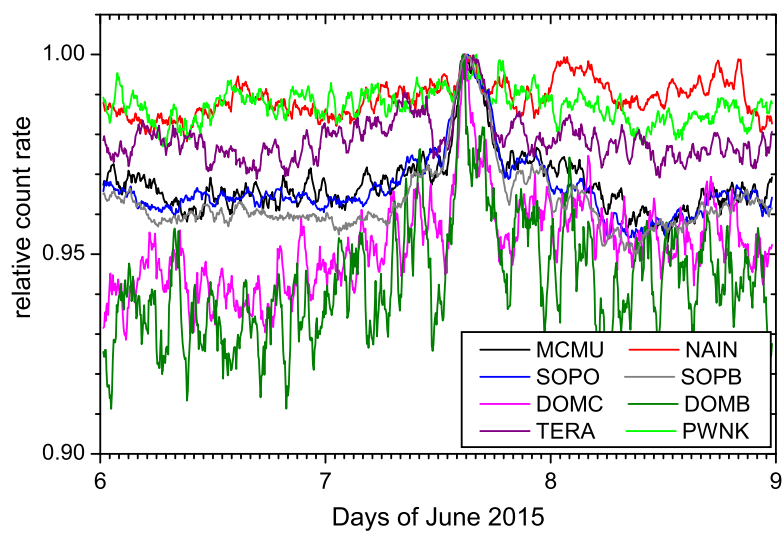

increase of $2-4 \%$. Thus, the significant signal was detected only by polar southern stations. In addition, two high-latitude northern-hemisphere NMs, Nain and Peawanuck, detected a marginal increase during the event that was statistically significant. We have also examined data from other available NMs for the period around the event and found no increases. 
Figure 3 Asymptotic directions of the analyzed NMs for the peak of the event (6 June 2017 15:00 UT). The upper panel shows the directions (in geographical coordinates) for protons in the rigidity range from $0.7-5 \mathrm{GV}$. The bottom panel shows the directions (in GSE coordinates) for $1 \mathrm{GV}$ rigidity protons. Stations with significant, marginal, and no increase are denoted as filled stars, open stars, and red crosses, respectively. Acronyms of station names are open in Table 1. The crossed circle corresponds to the direction of the IMF derived from the ACE satellite measurements during the event onset. The dotted curve represents the asymptotic cone for $1-1.5 \mathrm{GV}$ protons (the small black square corresponds to $1 \mathrm{GV}$ ) of the southernmost point of the PAMELA orbit at 14:34 UT.
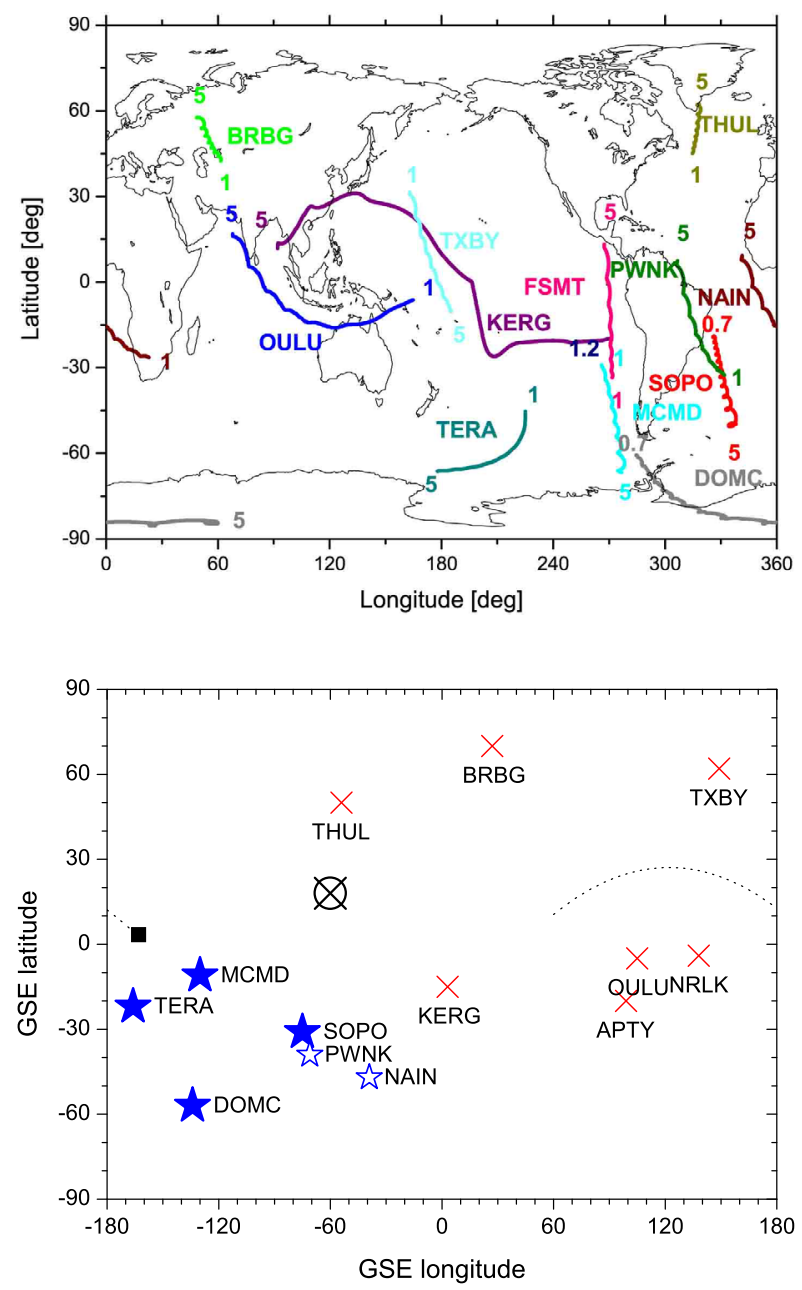

Physical location of an NM can be misleading because as a result of the bending of charged-particle trajectories in the Earth's magnetosphere, the directions of acceptance of charged particles by an NM may be quite far from its actual location (Smart, Shea, and Flückiger, 2000). For all the NMs we calculated the asymptotic viewing directions for the time of the event maximum (15:00 UT on 7 June 2015) by the standard method of particletrajectory backtracing described elsewhere (e.g. Mishev, Poluianov, and Usoskin, 2017). The asymptotic directions were computed using the PLANETOCOSMICS code (Desorgher et al., 2005) and applying the International Geomagnetic Reference Field (IGRF: Finlay et al., 2010), epoch 2015, and Tsyganenko-89 (Tsyganenko, 1989) as internal and external geomagnetic field models, respectively. The asymptotic directions (in geographical coordinates) for protons with rigidities from 0.7-5 GV are shown for some NMs in Figure 3 (top panel). In Figure 3 (bottom panel) we show the asymptotic directions in geocentric solar ecliptic (GSE) coordinates, corresponding to protons with a fixed rigidity of $1 \mathrm{GV}$, for a number of high-latitude NMs for the southern and northern hemispheres. Large blue stars correspond to stations with a significant response to the event, smaller blue open stars stand 
Figure 4 Near-Earth interplanetary conditions (hourly data) during the period of $6-8$ June 2015 according to the OMNIWeb Database. Top panel: integral proton flux, $F_{\mathrm{p}}$, with energies above 10,30 , and $60 \mathrm{MeV}$ as denoted in the legend. Central panel: solar wind proton flow speed. Bottom panel: magnitude of the total IMF. The gray hatched area denotes the interval of the event as identified from NMs.

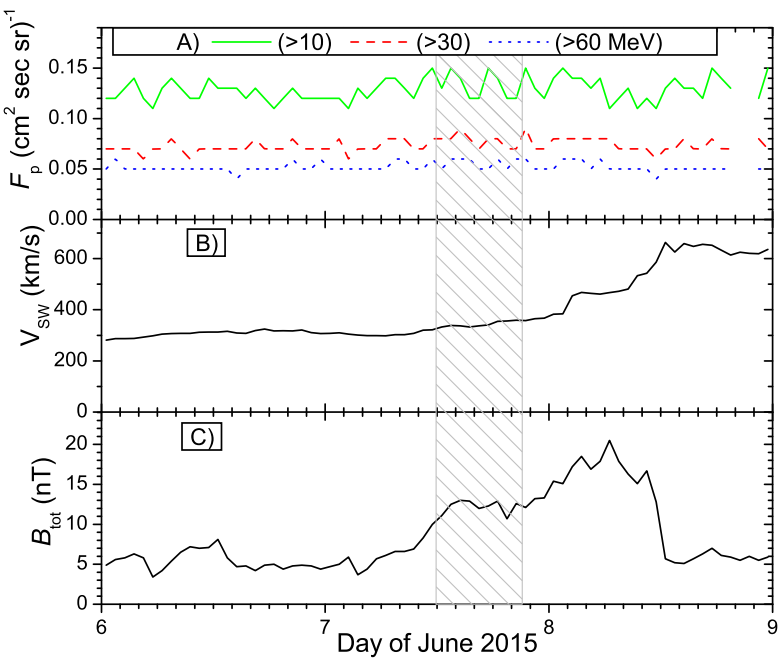

for the stations with a marginal response, and red crosses show stations with no response. It is interesting to observe that the asymptotic directions of the northern stations NAIN and PWNK are more south than that of the South Pole station. The significant response to the event is limited to a relatively small solid angle covering the range of about $60-180^{\circ}$ west longitude to the south (we emphasize that these are GSE, not geographical coordinates). It is important that this area is not related to the apparent direction of the interplanetary magnetic field (IMF), which is denoted as the black crossed circle for the time of the event maximum (according to data from the Advance Composition Explorer (ACE) satellite). There is no response in any other region, implying that the event was highly anisotropic, with the anisotropy direction being in the southwest sector and not aligned with the IMF direction.

\subsection{Space-Borne Data}

We have also checked available space-borne data for the same period.

Near-Earth data from the OMNIWeb database (https://omniweb.gsfc.nasa.gov/ow.html) imply the absence of any SEP event (top panels of Figure 4). The proton fluxes do not show any significant enhancement above the background variability during the time of the event in either energy range ( $>10,30$ or $60 \mathrm{MeV}$ ). There is a small enhancement of $>10 \mathrm{MeV}$ protons during the day of the event, but it is not different from the similar variability during other days.

Data from the Energetic and Relativistic Nuclei and Electron (ERNE: Torsti et al., 1995) detector on board the Solar and Heliospheric Observatory (SOHO) spacecraft are somewhat gappy during the period of interest, but fully consistent with the "no-response" background for the day of 7 June 2015 (Vainio, 2018, private communication). Since the instrument is located at the L1 point outside the magnetosphere, there is no need for geomagnetic correction.

We have also performed a careful search for a possible signal at the PAMELA detector (Adriani et al., 2014) for the date of the event. The PAMELA detector for studying cosmic rays flew during the period from June 2006 through January 2016 on board the Resurs-DK1 satellite on a polar orbit, which had a height of about $570 \mathrm{~km}$ in 2015 . Since the full analysis of the data from the PAMELA spectrometer in this low-energy range would not provide 
sufficient precision during a polar flyby, we used the count rate of its neutron detector as an integral index of cosmic-ray flux. The neutron detector consists of 18 pairs of helium-filled proportional counters ( $18.5 \mathrm{~mm}$ diameter and $209 \mathrm{~mm}$ length) surrounded by a polyethylene moderator and a cadmium layer that shield it from the slow neutrons that are produced in the satellite body. The detector permanently monitors the background neutron flux along the orbit, recording hits between triggers of the main spectrometer (Picozza et al., 2007). The count rate of the background neutron detector channel has a strong latitudinal dependence that varies between about 200 and $1000 \mathrm{~Hz}$ in equatorial and polar regions, respectively. The count rate is strongly enhanced in the region of the South Atlantic Anomaly (SAA) because of precipitation of the trapped particles from the radiation belt. The orbit moves longitudinally by about $26^{\circ}$ at each rotation, leading to a variation of the count rates that is related to the tilt of the geomagnetic field. Using the known dependence of the neutron-detector count rate on the geomagnetic cutoff rigidity, we have estimated the expected count rate along the orbit, which works well outside the SAA region. We compared the actually recorded values with the expected count rates for the day of 7 June 2015 and found no statistically significant difference. This implies that no detectable excess of secondary neutrons has been found that would correspond to the ACRE. Thus, data from the neutron counter of the PAMELA instrument are also consistent with a "no-response" background.

However, since PAMELA is a low-orbiting detector that is always inside the geomagnetosphere, an analysis of the asymptotic directions should be performed. The asymptotic directions of PAMELA are shown in Figure 3 for the southernmost point of its orbit at 14:34 UT on 7 June 2015, near the peak of the event. The asymptotic direction lies outside the event region as identified with NMs, thus confirming the anisotropic nature of the event. We note that the sensitivity of the PAMELA neutron counter to this low-energy particles is about $1 \%$ above the background, and a signal at a level of several percentages, as observed by Antarctic NMs, would have been statistically detected there if the detector had crossed the event region.

\section{Interplanetary Conditions}

The near-Earth interplanetary conditions during the event are shown in Figure 4: the integral flux of protons with energies above 10, 30, and $60 \mathrm{MeV}$ (top panel), plasma flow speed (central panel), and the IMF magnitude (bottom panel). The bottom panel of Figure 4 shows that the IMF strength started to grow from $5 \mathrm{nT}$ shortly before the event, at about 07:15 UT, until about noon of the next day, when it reached about $20 \mathrm{nT}$ and then suddenly dropped. This gradual increase was accompanied by a slow increase in solar wind speed from about $350 \mathrm{~km} \mathrm{~s}^{-1}$ before the event to about $690 \mathrm{~km} \mathrm{~s}^{-1}$ at noon of 8 June. This may indicate the presence of a moderate compression region that formed upstream of a high-speed wind stream. However, no severe change, such as an interplanetary shock, was observed. Accordingly, the heliospheric conditions were not greatly disturbed during the time of the event.

However, a large view shows that there was indeed a strong heliospheric disturbance that did not hit Earth, however, and thus was not measured in the near-Earth space in the ecliptic plane. A reconstruction (obtained from http://helioweather.net), using the ENLIL Model (Odstrčil, Smith, and Dryer, 1996) of the dynamical 3D heliospheric conditions is shown in Figure 5 for 14 UT of the day of the event. While the ecliptic plane was not much disturbed, there was a fast "ejecta" with a velocity of $600-700 \mathrm{~km} \mathrm{~s}^{-1}$. It passed south and west of the Earth, so that our planet was exactly at the boundary of the ejecta during the event. 

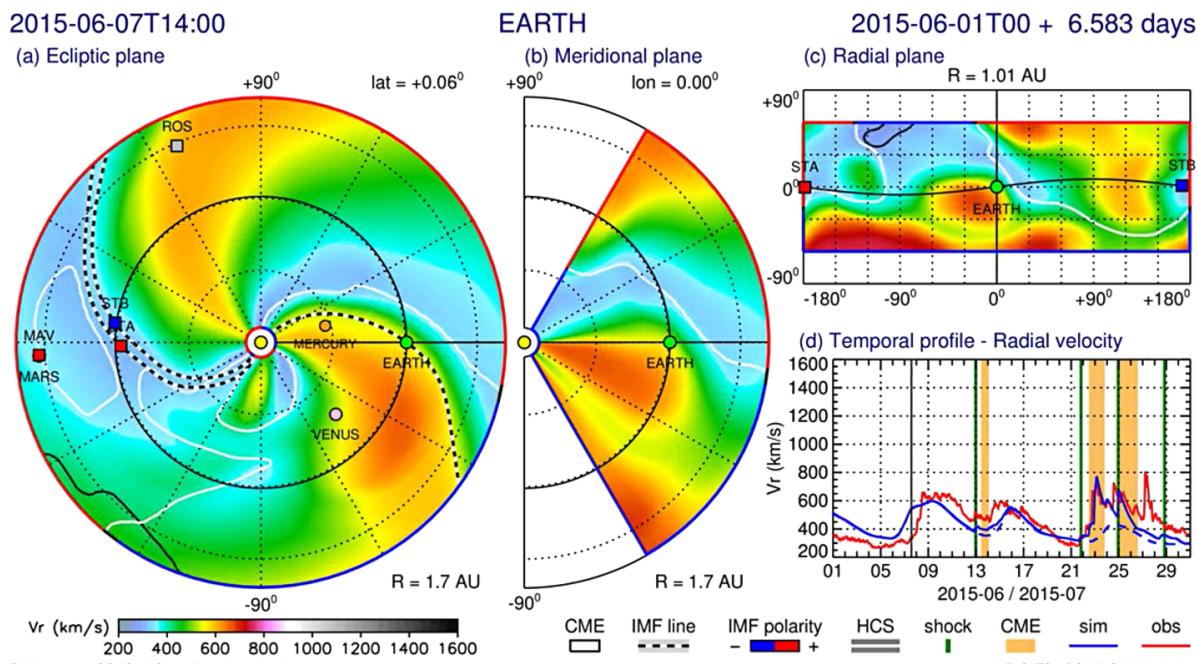
ENLIL-Iowres + GONGb.WSAlu/ tp400-d4×1/853h10

Figure 5 Solar wind radial velocity contour plots for 7 June 201514 UT shown in the ecliptic (a), meridional (b), and radial (c) planes. Panel $d$ shows the time profiles of the measured and simulated (red and blue, respectively) radial solar wind velocity near Earth. Simulation results are taken from http://helioweather.net.

\section{Discussion and Speculation}

We can summarize the experimental facts and speculate about the event as follows.

There was an increase in count rates of polar NMs, whose asymptotic directions lie in the southwest quadrant in GSE coordinates. The increase was strongest for the high-altitude nocutoff DOMB/DOMC station, moderate for the high-altitude low-cutoff SOPO/SOPB and the sea-level no-cutoff MCMD stations, and marginal for the low-cutoff sea-level stations NAIN and PWNK that are located in the northern hemisphere. This suggests that the effect was related to low-energy particles with a rigidity below $1 \mathrm{GV}$. No associated detectable increase was found in the space-borne data from the GOES, SOHO/ERNE, or PAMELA instruments. There are some hints, but they are not statistically significant. No SEP event occurred during the period in question.

The event took place on the background of relatively quiet near-Earth interplanetary conditions, without any strong disturbance as recorded by spacecraft in the ecliptic plane during the event. There was no Forbush decrease, in contrast to the event of 22 June 2015, nor any geomagnetic storm. However, a fast ejecta passed south and west of Earth, so that the planet was exactly at the boundary shear layer of the ejecta.

Based on an analysis of available data, we can speculate that the event was related to the fact that Earth brushed against a boundary layer between the high-speed stream and the slow solar wind region (Figure 5). The IMF strength and solar wind speed both gradually increased during the ACRE, corresponding to a compression region that was very asymmetric with respect to the ecliptic plane. This led to a strong local anisotropy of low-rigidity $(<1 \mathrm{GV})$ cosmic-ray flux. This event was similar to that of 22 June 2015 (and possibly also to the event on 18 January 2017), with the difference that the interplanetary disturbance did not hit Earth, but passed nearby. A more systematic study of this type of events is pending. We note that another apparently similar ACRE took place on 26 August 2018, after submission of this manuscript, and its full analysis is in progress. 
Acknowledgements Open access funding provided by University of Oulu including Oulu University Hospital. We thank Rami Vainio for his useful comments on the SOHO/ERNE data, and Dusan Odstrčil for valuable discussion on the heliospheric conditions. The work was partly supported by the projects of the Academy of Finland Centre of Excellence ReSoLVE (No. 272157), CRIPA, and CRIPA-X (No. 304435), and by the Finnish Antarctic Research Program (FINNARP). A.G. acknowledges the Polish National Science Centre, decision number DEC-2016/22/E/HS5/00406. V.M. acknowledges the Ministry of Education and Science of the Russian Federation (contract no. 3.2131.2017). European Neutron Monitor database NMDB (http://nmdb.eu) and PI of individual NMs are acknowledged for cosmic-ray data. Data from the OULU, DOMC, and DOMB stations are directly available at http://cosmicrays.oulu.fi. The neutron monitor data from South Pole are provided by the University of Wisconsin, River Falls. Collaboration and staff of the French-Italian Concordia Station (IPEV program n903 and PNRA Project LTCPAA PNRA14_00091) is acknowledged for support of DOMB/DOMC monitors. Simulation results are from http://helioweather.net and also have been provided by the Community Coordinated Modeling Center at Goddard Space Flight Center through their public Runs on Request system (http://ccmc.gsfc.nasa.gov). The ENLIL Model was developed by Dusan Odstrčil, now at the George Mason University - Space Weather Lab and NASA/GSFC - Space Weather Lab.

Disclosure of Potential Conflicts of Interest The authors declare that they have no conflicts of interest.

Open Access This article is distributed under the terms of the Creative Commons Attribution 4.0 International License (http://creativecommons.org/licenses/by/4.0/), which permits unrestricted use, distribution, and reproduction in any medium, provided you give appropriate credit to the original author(s) and the source, provide a link to the Creative Commons license, and indicate if changes were made.

\section{References}

Adriani, O., Barbarino, G.C., Bazilevskaya, G.A., Bellotti, R., Boezio, M., Bogomolov, E.A., Bongi, M., Bonvicini, V., Bottai, S., Bruno, A., Cafagna, F., Campana, D., Carbone, R., Carlson, P., Casolino, M., Castellini, G., De Pascale, M.P., De Santis, C., De Simone, N., Di Felice, V., Formato, V., Galper, A.M., Giaccari, U., Karelin, A.V., Kheymits, M.D., Koldashov, S.V., Koldobskiy, S., Krut'kov, S.Y., Kvashnin, A.N., Leonov, A., Malakhov, V., Marcelli, L., Martucci, M., Mayorov, A.G., Menn, W., Mikhailov, V.V., Mocchiutti, E., Monaco, A., Mori, N., Munini, R., Nikonov, N., Osteria, G., Papini, P., Pearce, M., Picozza, P., Pizzolotto, C., Ricci, M., Ricciarini, S.B., Rossetto, L., Sarkar, R., Simon, M., Sparvoli, R., Spillantini, P., Stozhkov, Y.I., Vacchi, A., Vannuccini, E., Vasilyev, G.I., Voronov, S.A., Wu, J., Yurkin, Y.T., Zampa, G., Zampa, N., Zverev, V.G.: 2014, The PAMELA Mission: Heralding a new era in precision cosmic ray physics. Phys. Rep. 544, 323. DOI. ADS.

Ahluwalia, H.S., Fikani, M.M.: 2007, Cosmic ray detector response to transient solar modulation: Forbush decreases. J. Geophys. Res. 112, A08105. DOI. ADS.

Cane, H.V.: 2000, Coronal mass ejections and Forbush decreases. Space Sci. Rev. 93, 55. DOI. ADS.

Desai, M., Giacalone, J.: 2016, Large gradual solar energetic particle events. Living Rev. Solar Phys. 13, 3. DOI. ADS.

Desorgher, L., Flückiger, E.O., Gurtner, M., Moser, M.R., Bütikofer, R.: 2005, Atmocosmics: A geant 4 code for computing the interaction of cosmic rays with the Earth's atmosphere. Int. J. Mod. Phys. A 20, 6802. DOI. ADS.

Finlay, C.C., Maus, S., Beggan, C.D., Bondar, T.N., Chambodut, A., Chernova, T.A., Chulliat, A., Golovkov, V.P., Hamilton, B., Hamoudi, M., Holme, R., Hulot, G., Kuang, W., Langlais, B., Lesur, V., Lowes, F.J., Lühr, H., MacMillan, S., Mandea, M., McLean, S., Manoj, C., Menvielle, M., Michaelis, I., Olsen, N., Rauberg, J., Rother, M., Sabaka, T.J., Tangborn, A., Tøffner-Clausen, L., Thébault, E., Thomson, A.W.P., Wardinski, I., Wei, Z., Zvereva, T.I.: 2010, International geomagnetic reference field: The eleventh generation. Geophys. J. Int. 183, 1216. DOI. ADS.

Jokipii, J.R., Levy, E.H.: 1977, Effects of particle drifts on the solar modulation of galactic cosmic rays. Astrophys. J. Lett. 213, L85. DOI. ADS.

Klein, K.-L., Dalla, S.: 2017, Acceleration and propagation of solar energetic particles. Space Sci. Rev. 212, 1107. DOI. ADS.

Kudela, K., Bučík, R., Bobík, P.: 2008, On transmissivity of low energy cosmic rays in disturbed magnetosphere. Adv. Space Res. 42, 1300. DOI. ADS.

Lockwood, J.A.: 1971, Forbush decreases in the cosmic radiation. Space Sci. Rev. 12, 658. DOI. ADS.

Mangeard, P.-S., Ruffolo, D., Sáiz, A., Madlee, S., Nutaro, T.: 2016, Monte Carlo simulation of the neutron monitor yield function. J. Geophys. Res. 121, 7435. DOI. ADS. 
Mangeard, P.-S., Muangha, P., Pyle, R., Ruffolo, D., Sàiz, A., et al. (The IceCube collaboration): 2017, Impulsive increase of galactic cosmic ray flux observed by icetop. In: Proc. 35th Internat. Cosmic Ray Conf. 35, 133.

Mavromichalaki, H., Papaioannou, A., Plainaki, C., Sarlanis, C., Souvatzoglou, G., Gerontidou, M., Papailiou, M., Eroshenko, E., Belov, A., Yanke, V., Flückiger, E.O., Bütikofer, R., Parisi, M., Storini, M., Klein, K.-L., Fuller, N., Steigies, C.T., Rother, O.M., Heber, B., Wimmer-Schweingruber, R.F., Kudela, K., Strharsky, I., Langer, R., Usoskin, I., Ibragimov, A., Chilingaryan, A., Hovsepyan, G., Reymers, A., Yeghikyan, A., Kryakunova, O., Dryn, E., Nikolayevskiy, N., Dorman, L., Pustil'Nik, L.: 2011, Applications and usage of the real-time Neutron Monitor Database. Adv. Space Res. 47, 2210. DOI. ADS.

Mishev, A., Poluianov, S., Usoskin, I.: 2017, Assessment of spectral and angular characteristics of sub-GLE events using the global neutron monitor network. J. Space Weather Space Clim. 7(27), A28. DOI. ADS.

Mohanty, P.K., Arunbabu, K.P., Aziz, T., Dugad, S.R., Gupta, S.K., Hariharan, B., Jagadeesan, P., Jain, A., Morris, S.D., Rao, B.S., Hayashi, Y., Kawakami, S., Oshima, A., Shibata, S., Raha, S., Subramanian, P., Kojima, H.: 2016, Transient weakening of Earth's magnetic shield probed by a cosmic ray burst. Phys. Rev. Lett. 117, 171101. DOI.

Munakata, K., Kozai, M., Evenson, P., Kuwabara, T., Kato, C., Tokumaru, M., Rockenbach, M., Dal Lago, A., de Mendonca, R.R.S., Braga, C.R., Schuch, N.J., Jassar, H.K.A., Sharma, M.M., Duldig, M.L., Humble, J.E., Sabbah, I., Kóta, J.: 2018, Cosmic-ray short burst observed with the Global Muon Detector Network (GMDN) on 2015 June 22. Astrophys. J. 862, 170. DOI. ADS.

Odstrčil, D., Smith, Z., Dryer, M.: 1996, Distortion of the heliospheric plasma sheet by interplanetary shocks. Geophys. Res. Lett. 23, 2521. DOI. ADS.

Picozza, P., Galper, A.M., Castellini, G., Adriani, O., Altamura, F., Ambriola, M., Barbarino, G.C., Basili, A., Bazilevskaja, G.A., Bencardino, R., Boezio, M., Bogomolov, E.A., Bonechi, L., Bongi, M., Bongiorno, L., Bonvicini, V., Cafagna, F., Campana, D., Carlson, P., Casolino, M., de Marzo, C., de Pascale, M.P., de Rosa, G., Fedele, D., Hofverberg, P., Koldashov, S.V., Krutkov, S.Y., Kvashnin, A.N., Lund, J., Lundquist, J., Maksumov, O., Malvezzi, V., Marcelli, L., Menn, W., Mikhailov, V.V., Minori, M., Misin, S., Mocchiutti, E., Morselli, A., Nikonov, N.N., Orsi, S., Osteria, G., Papini, P., Pearce, M., Ricci, M., Ricciarini, S.B., Runtso, M.F., Russo, S., Simon, M., Sparvoli, R., Spillantini, P., Stozhkov, Y.I., Taddei, E., Vacchi, A., Vannuccini, E., Voronov, S.A., Yurkin, Y.T., Zampa, G., Zampa, N., Zverev, V.G.: 2007, PAMELA A payload for antimatter matter exploration and light-nuclei astrophysics. Astropart. Phys. 27, 296. DOI. ADS.

Poluianov, S., Usoskin, I., Mishev, A., Moraal, H., Kruger, H., Casasanta, G., Traversi, R., Udisti, R.: 2015, Mini neutron monitors at Concordia research station, Central Antarctica. J. Astron. Space Sci. 32, 281. DOI. ADS.

Potgieter, M.: 2013, Solar modulation of cosmic rays. Living Rev. Solar Phys. 10, 3. DOI. ADS.

Simpson, J.A.: 2000, The cosmic ray nucleonic component: The invention and scientific uses of the neutron monitor - (keynote lecture). Space Sci. Rev. 93, 11. DOI. ADS.

Smart, D.F., Shea, M.A., Flückiger, E.O.: 2000, Magnetospheric models and trajectory computations. Space Sci. Rev. 93, 305. DOI. ADS.

Torsti, J., Valtonen, E., Lumme, M., Peltonen, P., Eronen, T., Louhola, M., Riihonen, E., Schultz, G., Teittinen, M., Ahola, K., Holmlund, C., Kelhä, V., Leppälä, K., Ruuska, P., Strömmer, E.: 1995, Energetic particle experiment ERNE. Solar Phys. 162, 505. DOI. ADS.

Tsyganenko, N.A.: 1989, A magnetospheric magnetic field model with a warped tail current sheet. Planet. Space Sci. 37, 5. DOI.

Usoskin, I.G., Bazilevskaya, G.A., Kovaltsov, G.A.: 2011, Solar modulation parameter for cosmic rays since 1936 reconstructed from ground-based neutron monitors and ionization chambers. J. Geophys. Res. 116, A02104. DOI. ADS.

Usoskin, I., Poluianov, S., Moraal, H., Krüger, H., Casasanta, G., Traversi, R., Udisty, R.: 2015, A mini neutron monitor in Central Antarctica (Dome Concordia). In: Borisov, A.S., Denisova, V.G., Guseva, Z.M., Kanevskaya, E.A., Kogan, M.G., Morozov, A.E., Puchkov, V.S., Pyatovsky, S.E., Shoziyoev, G.P., Smirnova, M.D., Vargasov, A.V., Galkin, V.I., Nazarov, S.I., Mukhamedshin, R.A. (eds.) Proc. 34th Internat. Cosmic Ray Conf., 217. ADS.

Vainio, R., Desorgher, L., Heynderickx, D., Storini, M., Flückiger, E., Horne, R.B., Kovaltsov, G.A., Kudela, K., Laurenza, M., McKenna-Lawlor, S., Rothkaehl, H., Usoskin, I.G.: 2009, Dynamics of the Earth's particle radiation environment. Space Sci. Rev. 147, 187. DOI. 